\title{
1 Functional poly(2-oxazoline)s by direct amidation of methyl ester side chains
}

${ }^{1}$ Supramolecular Chemistry Group, Department of Organic and Macromolecular Chemistry,

7 Ghent University, Krijgslaan 281 S4, B-9000 Ghent Belgium; richard.hoogenboom@ugent.be

11 KEYWORDS: post polymerization modification, poly(2-oxazoline)s, hydrolysis, poly(ethylene

12 imine), coupling reaction, uncatalyzed amidation, biocompatible polymers 
2 ABSTRACT Poly(2-alkyl/aryl-2-oxazoline)s (PAOx) are biocompatible pseudo-polypeptides that

3 received significant interest for biomedical applications in recent years. The growing popularity

4 of PAOx in recent years is driven by its much higher chemical versatility compared to the gold

5 standard in this field, poly(ethylene glycol) (PEG), while having similar beneficial properties, such

6 as Stealth behaviour and biocompatibility. In this article, we further expand the PAOx chemical

7 toolbox by demonstrating a novel straightforward and highly versatile post-polymerization

8 modification platform for the introduction of side-chain functionalities. PAOx having side chain

9 methyl ester functionalities are demonstrated to undergo facile uncatalyzed amidation reactions

10 with a wide range of amines yielding the corresponding PAOx with side chain secondary amide

11 groups containing short aliphatic linkers as well as a range of side-chain functionalities including

12 acid, amine, alcohol, hydrazide and propargyl groups. The PAOx with side chain methyl ester

13 groups can be either prepared by partial hydrolysis of a PAOx followed by introduction of the

14 methyl ester via modification of the secondary amine groups with methyl succinylchloride or by

15 direct copolymerization of a non-functional 2-oxazoline monomer with a 2-

16 methoxycarbonylethyl-2-oxazoline. Thus, this novel synthetic platform enable direct access to a

17 wide range of side-chain functionalities from the same methyl ester functionalized poly(2-

18 oxazoline) scaffold. 


\section{Introduction}

3 the cationic ring opening polymerization (CROP) of 2-aryl-2-oxazolines and 2-alkyl-2-

4 oxazolines, such as 2-phenyl-2-oxazoline and 2-methyl-2-oxazoline. ${ }^{1-4}$ After this discovery, the

5 CROP of 2-oxazolines has gained significant interest. Well-defined poly(2-alkyl/aryl-2-

6 oxazoline)s (PAOx) can be obtained if the polymerizations are done under nucleophile free

7 conditions to avoid side reactions such as chain transfer and termination. ${ }^{5,6}$ After the flourishing

8 of PAOx in the seventies and eighties, research interest was retracting in the nineties. Since the

9 dawn of the new millennium, PAOx are quickly gaining interest again, as researchers have

10 discovered their high potential for biomedical applications. ${ }^{7-10}$ In addition, a microwave assisted

11 polymerization protocol was introduced about ten years ago to drastically reduce polymerization

12 times from days and hours to minutes. ${ }^{11,12}$ The livingness of the CROP allows the preparation

13 of defined PAOx with full control over polymer architectures including blocks ${ }^{13,14}$, gradients ${ }^{15}$

14 and star-shaped structure ${ }^{16}$. Furthermore the properties of PAOx are highly tunable by variation

15 of the side-chain R group as well as by copolymerization of different monomers. ${ }^{17-19}$

16 The current interest in PAOx is more and more shifting towards biomedical applications

17 stimulated by the high biocompatibility and stealth behaviour, which is especially documented

18 for poly(2-methyl-2-oxazoline) $(\mathrm{PMeOx})$ and poly(2-ethyl-2-oxazoline) (PEtOx). ${ }^{7,20}$ In fact,

19 they can arguably compete or even outperform the gold standards of the field, namely

20 poly(ethylene glycol) and poly( $N$-hydroxypropylmethacrylamide) ${ }^{21-24}$ For use of PAOx in

21 biomedical applications, it is important to have easy and straightforward functionalization

22 methodologies, either for conjugation of the polymers to biological media or substrates or to

23 attach drug molecules, labels or targeting moieties. Up to three orthogonal functionalities can be 
1 (simultaneously) introduced in PAOx through initiation, termination and incorporation of

2 functional monomers. ${ }^{18,25}$ The choice of functional monomers is however limited to groups that

3 are non-nucleophilic as any nucleophile will interfere with the polymerization process.

4 A major advantage of PAOx over PEG is the possibility to introduce functionalities in the side

5 chains by simple copolymerization of a functional monomer with a non-functional monomer.

6 However, many functional groups need to be protected during the CROP to avoid interference

7 with the polymerization conditions, as has been reported for monomers with acid ${ }^{12,19,26}$,

8 alcohol $^{19}$, amine ${ }^{12}$, thiol ${ }^{19}$ and aldehyde ${ }^{19,27,28}$ functionalities. An important exception are

9 monomers containing double and triple bonds that are not affected by the living CROP. The

10 direct introduction of internal and terminal double bonds as well as terminal triple bonds into

11 PAOx has been demonstrated ${ }^{14,27,29,30}$. These polymers could further be utilized for efficient

12 post-polymerization modification by thiol-ene ${ }^{25,31,32}$, thiol-yne ${ }^{32}$ and copper(I) catalysed azide-

13 alkyne cycloaddion (CuAAC) $)^{31,32}$, so called click reactions ${ }^{33}$.

14 In this work, we report a novel straightforward post-polymerization modification strategy for

15 PAOx, which allows the incorporation of a wide range of side-chain functionalities by direct

16 amidation of the side chain methyl ester groups with different amines as shown in Scheme 1. The

17 PAOx building blocks containing side-chain methyl ester groups can either be prepared by partial

18 hydrolysis of PAOx followed by introduction of the methyl ester functionality by reaction with

19 methyl succinyl chloride or by copolymerization of a non-functional 2-oxazoline monomer with

20 2-methoxycarbonylethyl-2-oxazoline ${ }^{4,34}$. Within this manuscript we will demonstrate the

21 versatility of this new synthetic methodology. 

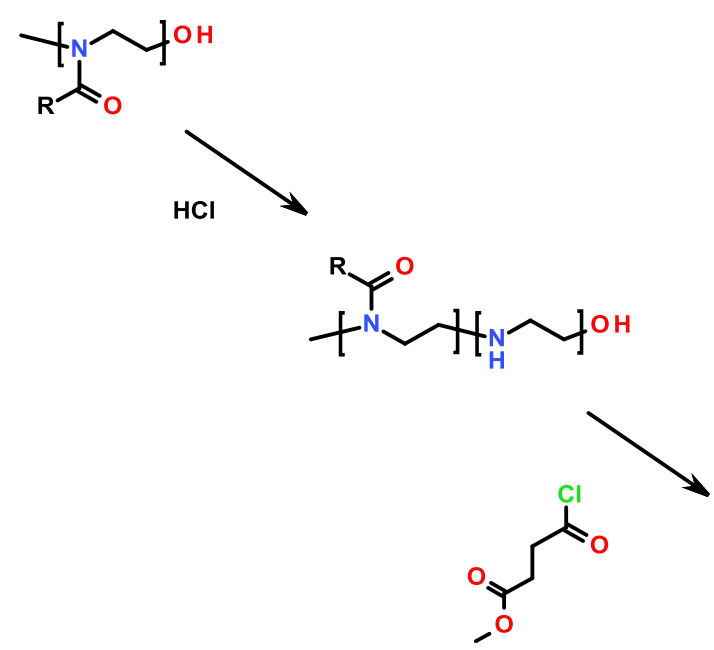

2 Scheme 1. Schematic representation of the partial hydrolysis of a poly(2-oxazoline) followed by

3 modification with methyl succinyl chloride and finally side-chain modification by direct amidation

4 with an amine. The direct synthesis of the methyl ester containing PAOx by copolymerization is 5 also included in the scheme as alternative route ${ }^{34}$.

\section{Experimental Section}

11 dichloromethane, aminomethane, aminoethane, 1-aminopropane, dimethylamine, hydrazine,

12 glycine, Leucine methyl ester, 1,3 aminopropanol, allylamine, 1-amino-3-butyn, allylamine, N,N'-

13 dimethyl-1,2-ethyleene diamine, 1,2 ethylene diamine, methyl succinyl chloride and amino 
1 ethanol were obtained from Sigma Aldrich and used as received. ${ }^{1} \mathrm{H}$ NMR and ${ }^{13} \mathrm{C}$ NMR spectra

2 were recorded in $\mathrm{CDCl}_{3}$ or in DMSO on a Bruker Avance $300 \mathrm{MHz}$ or $500 \mathrm{MHz}$ spectrometer.

3 Size exclusion chromatography (SEC) was performed on a Agilent HPLC with a 1260 refractive

4 index detector (RID) using dimethylacetamide containing $50 \mathrm{mM} \mathrm{LiCl}$ as eluent at a flow rate of

$50.6 \mathrm{ml} / \mathrm{min}$. Polymethylmethacrylate (PMMA) standards were used to calculate the molar mass

6 values and the column set consisted of two PLgel $5 \mu \mathrm{m}$ mixed D columns at $40^{\circ} \mathrm{C}$ and a similar

7 guard column (Agilent) in series. Chromatograms were analyzed using Agilent Chemstation

8 software with the GPC add on. The polymers obtained after amidation were analyzed by size

9 exclusion chromatography (HFIP-SEC) using a Agilent HPLC with a 1260 refractive index

10 detector (RID) with eluent of hexafluoro-2-propanol(HFIP) containing $20 \mathrm{mM}$ Sodium

11 trifluoroacetate at a flow rate of $0.3 \mathrm{ml} / \mathrm{min}$. Polymethylmethacrylate (PMMA) standards were

12 used to calculate the molar mass values and the column set consisted of two PSS PFG $100 \AA$ gel 5

$13 \mu \mathrm{m}$ mixed $\mathrm{D}$ columns and a similar guard column (Agilent) at $35^{\circ} \mathrm{C}$ in series Chromatograms were

14 analyzed using Agilent Chemstation software with the GPC add on. The FT-IR was performed on

15 a Perkin Elmer 1000 FTIR spectrum meter with PIKE HATR module. All of the polymerization

16 where done in a Vigor glovebox. Matrix assisted laser desorption/ionization time of flight mass

17 spectroscopy (MALDI-TOF MS) was performed on an Applied Biosystems Voyager De STR

18 MALDI-TOF mass spectrometer equipped with $2 \mathrm{~m}$ linear and $3 \mathrm{~m}$ reflector flight tubes, and a

$19355 \mathrm{~nm}$ Blue Lion Biotech Marathon solid state laser (3.5 ns pulse). All mass spectra were obtained

20 with an accelerating potential of $20 \mathrm{kV}$ in positive ion mode and in reflectron mode.2-(4'-

21 Hydroxybenzeneazo)benzoic acid (HABA) $(20 \mathrm{mg} / \mathrm{ml}$ in acetone) was used as matrix. Polymer

22 samples were dissolved in acetone $(2 \mathrm{mg} / \mathrm{ml})$. Analyte solutions were prepared by mixing $10 \mu \mathrm{l}$

23 matrix and $5 \mu$ polymer samples. Samples were applied using the dried droplet method. 
2 Partial hydrolysis of PEtOx. The poly-(2-ethyl-2-oxazoline) ((10 g) was dissolved in $50 \mathrm{ml}$ of 3 water with heating and stirring until everything was dissolved. Then $50 \mathrm{ml}$ of $36 \%$ solution of $\mathrm{HCl}$

4 was added and the mixture was heated to $73^{\circ} \mathrm{C}$ (internal temperature) for $200 \mathrm{~min}$. After the 5 volatiles were removed on a rotary evaporator, the polymer was redissolved in water and the 6 solution was basidified to $\mathrm{pH} 10-11$ using a $\mathrm{NaOH}$ solution. The solution was then lyophilized and 7 the solid was dissolved in dichloromethane. The organic phase was washed with brine and then 8 concentrated in vacuum to yield the PEtOx-PEI as a solid white product ( $7 \mathrm{~g} ; 77 \%$ yield $).{ }^{1} H$-NMR

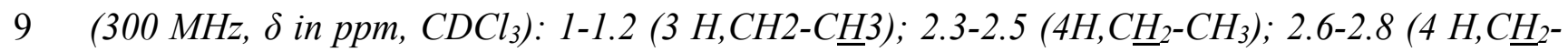
$10 \mathrm{NH}) ; 3.2-3.5(4 \mathrm{H}, \mathrm{CH} 2 \mathrm{NCH} 2)$. The integral ratios between the peaks at 2.6-2.8 ppm and 3.2-3.5 11 ppm were utilized to calculate the degree of hydrolysis to be $18 \%$.

13 Modification of PEtOx-PEI with methyl succinyl chloride. $7 \mathrm{~g}$ of the PEtOX-PEI (18\%) was

14 several times co-evaporated with toluene to remove any water. Subsequently, the polymer was 15 dissolved in dry dichloromethane $\left(6 \mathrm{ml} / \mathrm{g}\right.$ of polymer) and the mixture was cooled to $0^{\circ} \mathrm{C}$. Methyl 16 succinyl chloride (4.5 ml, $3.65 \mathrm{mmol}, 2$ eq.) was added to this solution of PEtOx-PEI followed by 17 drop wise addition of triethylamine (2 eq, $0.3 \mathrm{ml})$. The mixture was stirred for $24 \mathrm{~h}$ after which the 18 reaction mixture was directly precipitated in cold ether. The precipitated polymer was isolated by 19 filtration and dried under vacuum to yield the PEtOx copolymer with methyl ester side chains 20 (PEtOx-MestOx) in 84\% yield (7.5 g). ${ }^{1} \mathrm{H}-\mathrm{NMR}\left(300 \mathrm{MHz}\right.$, $\delta$ in $\left.\mathrm{ppm}, \mathrm{CDCl}_{3}\right): 1-1.2\left(3 \mathrm{H}, \mathrm{CH}_{2^{-}}\right.$ $\left.21 \mathrm{C} \underline{H}_{3}\right) ; 2.3-2.5\left(4 H, \underline{C H}_{2}-C H_{3}\right) ; 2.6-2.8\left(4 \mathrm{H}, \underline{C H}_{2}-\mathrm{NH}\right) ; 3.2-3.5\left(4 \mathrm{H}, \mathrm{C} \underline{H}_{2} \mathrm{~N}-\mathrm{CH} 2\right) ; 3.7$ (3 H,O-C $\left.\underline{H}_{3}\right)$.

22 SEC-data $M_{w} 1.7610^{4} ; M_{n} 1.6710^{4}$ and $Đ 1.1$. The IR spectrum showed following peaks $\left(\mathrm{cm}^{-1}\right)$ : $231731\left(\mathrm{CH}_{3}-\mathrm{O}\right.$, ester ) 1630 (all amides). 
2 Amine coupling method 1: (aminomethane, aminoethane, 1-aminopropane, hydrazine). The

3 PEtOx-PMestOx copolymer (300 mg, 4.6*10-4 mol) was directly dissolved in an excess of amine

$4 \quad(\sim 6 \mathrm{ml})$. The reaction was stirred for 2 days at $70^{\circ} \mathrm{C}$ after which the solution was evaporated to

5 dryness. Purification was done using preparative SEC, PD 10 column with water ass eluent, to

6 yield the modified polymers in near-quantitative yields.

8 Amine coupling method 2: (1-amino-2-ethanol, 1-aminopropanol, allylamine, amino-3-

9 butyne, $\mathbf{N}, \mathbf{N}$-dimethylethylene diamine). The PEtOx-PMestOx (300 mg,4.6*10-4 mol) copolymer

10 was dissolved in $6 \mathrm{ml}$ of acetonitrile; for amines that are not soluble in acetonitrile the solvent was

11 changed to DMF. In the case where the amines where part of on an amino acid, such as glycine or

12 Leucine, water with a $\mathrm{pH}$ of 9 was used to ensure that all amines where deprotonated. A

13 concentrated solution of amine ( 3 equivalents to the methyl ester) was then added and the reaction

14 mixture was stirred for 1 day at $40^{\circ} \mathrm{C}$. After evaporation of the solution under reduced pressure,

15 the remaining polymer was purified by preparative SEC, PD 10 column with water as eluent

16 yielding the modified polymers in near-quantitative yields.

18 Polymerisation of the methyl ester functionalized monomer(MestOx) DP 20. The MestOx

19 monomer was synthesised as described earlier using methyl tosylate as an initiator. ${ }^{34}$ The

20 polymerisation was carried out in a $20 \mathrm{ml}$ microwave vial. The methyl tosylate $(0.34 \mathrm{ml} ; 0.002$

$21 \mathrm{~mol})$ was dissolved in a solution containing $7 \mathrm{~g}$ of MestOx monomer( $7 \mathrm{~g} ; 0.04 \mathrm{~mol})$ and $8 \mathrm{ml}$ of

22 acetonitrile obtaining a $3 \mathrm{M}$ polymer solution. The reaction mixture was prepared in the glovebox

23 ensuring an oxygen and water free environment. The polymerisation was performed by heating 
1 the mixture for 2.35 minutes to $140{ }^{\circ} \mathrm{C}$ in the microwave. After polymerisation the reaction was

2 terminated by addition of $1 \mathrm{ml}$ of a $1 \mathrm{M}$ solution $\mathrm{KOH}$ in methanol. The acetonitrile was then

3 removed via reduced pressure and the polymer was dissolved in dichloromethane and precipitated

4 in cold ether(10-fold excess). The polymer was then dissolved in water and freeze dried to obtain

5 a white powder (3.311g; yield 65\%). ${ }^{1} \mathrm{H}-\mathrm{NMR}\left(500 \mathrm{MHz}\right.$, $\delta$ in ppm, $\left.\mathrm{D}_{2} \mathrm{O}\right): ; 2.5-2.7(4 \mathrm{H}, \mathrm{CO}-\mathrm{CH}-$

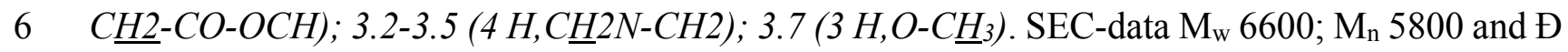

$7 \quad 1.12$.

9 Results and discussions

10 Synthesis of PEtOx-MestOx via hydrolysis and modification of PEtOx

11 To evaluate the newly proposed post-polymerization modification platform of PAOx copolymers

12 with methyl ester side chains with amines (Scheme 1), a well-defined PEtOx was first prepared as

13 starting material, whereby the narrow dispersity (Đ) will facilitate the evaluation of the products

14 after each reaction step by SEC. Therefore, a large batch (10 g) of PEtOx with a DP of 100 was

15 prepared using the previously optimized microwave assisted polymerization protocol yielding

16 well-defined PEtOx with a $Đ$ of 1.1 determined by SEC with DMA as eluent. ${ }^{11}$

17 Next, the well-defined PEtOx was hydrolyzed in controlled acidic conditions and based on the

18 previously established hydrolysis kinetics, a good control can be achieved over the hydrolysis

19 rate. ${ }^{35}$ This allows for an easy control over the amount of secondary amines in PEtOx-PEI and,

20 thus, provides control over the amount of methyl ester functional groups that can be attached to

21 the main chain. To aim for a hydrolysis degree around $20 \%$ the hydrolysis was performed in $\sim 6.8$

$22 \mathrm{~N}$ hydrochloric acid at $73^{\circ} \mathrm{C}$ for $200 \mathrm{~min}$. The degree of hydrolysis was determined by ${ }^{1} \mathrm{H}-\mathrm{NMR}$

23 spectroscopy. The integral ratio of signals corresponding to the backbone of PEtOx at $\delta 3.5 \mathrm{ppm}$ 
1 and the backbone of PEI at $\delta 2.8 \mathrm{ppm}$ was used to calculate the degree of hydrolysis, as shown in

2 ${ }^{1} \mathrm{H}-\mathrm{NMR}$. It is shown that $18 \%$ of the PEtOx units were hydrolysed yielding a $\mathrm{PEtO}_{82}-\mathrm{PEI}_{18}$ 3 copolymer. Purification of this PEtOx-PEI was done by first drying the reaction mixture on a rotary

4 evaporator to remove the water, propionic acid and excess of hydrochloric acid. Subsequently the 5 polymer is dissolved in a small amount of water and the $\mathrm{pH}$ is adjusted to $\mathrm{pH} 10-11$ with $\mathrm{NaOH}$, 6 to deprotonate the PEI units, followed by lyophilization. The resulting white powder is 7 subsequently dissolved in dichloromethane and extracted with brine and water to remove the traces 8 of propionate as well as the $\mathrm{NaOH}$. The final purified polymer is collected by evaporation of the 9 dichloromethane. This partially hydrolyzed copolymer can no longer by analysed by SEC in DMA 10 due to too strong interactions between the amine groups and the column material leading to tailing 11 of the signal and with higher hydrolysis degrees even to complete sticking of the polymer to the 12 column. Therefore, SEC of PEtOx-PEI was measured with hexafluoroisopropanol (HFIP) as 13 eluent ${ }^{36}$, revealing a $\mathrm{M}_{\mathrm{n}}$ of $1.510^{4} \mathrm{Da}$ and $\mathrm{Ð}$ of 1.3. The broadening of size distribution can be 14 ascribed to the SEC system and column set as the PEtOx starting polymer revealed a $M_{n}$ of $7.610^{3}$ 15 Da and a $Đ$ of 1.4 on this SEC system with HFIP. The counterintuitive increase in $M_{n}$ upon removal 16 of $18 \%$ of the side chains is in line with previous observations and can be ascribed to the larger 17 hydrodynamic volume of the partially hydrolyzed copolymer in HFIP, presumably due to better 18 hydrogen bonding of the HFIP to the polymer chains.

19 The prepared PEtOx-PEI represents a well-defined reaction platform containing the 20 biocompatible PEtOx as main structure together with the nucleophilic secondary amine units from 21 the PEI. A wide variety of modification reactions of such partially hydrolysed polymers have 22 already been reported in literature ${ }^{37}$. In this work, the PEtOx-PEI copolymer was reacted with 23 methyl succinylchloride to reintroduce a side chain amide group similar to the native PAOx 
1 structure containing a latent methyl ester functionality yielding PEtOx ${ }_{82} \mathrm{MestOx}_{18}$. The ${ }^{1} \mathrm{H}-\mathrm{NMR}$

2 spectrum of PEtOx-MestOx revealed the disappearance of the PEI backbone resonances at 2.6

$3 \mathrm{ppm}$ as well as the appearance of the methyl ester peak at $3.7 \mathrm{ppm}$ demonstrating the success of

4 the modification reaction. Furthermore, the IR spectrum also revealed the representative methyl

5 ester carbonyl vibration at $1730 \mathrm{~cm}^{-1}$. The SEC trace of PEtOx-PMestOx in HFIP also revealed a

6 decrease in $\mathrm{M}_{\mathrm{n}}$ caused by the change in hydrodynamic volume while the $\bigoplus$ remained the same ( $Ð$

7 = 1.3). Overlays of the SEC traces and ${ }^{1} \mathrm{H}$ NMR spectra of PEtOx, PEtOx-PEI and PEtOx-MestOx

8 are shown in Figure 1 to illustrate the successful modifications.

9 As mentioned in the introduction PEtOx-MestOx can also be obtained by direct 10 copolymerization of 2-ethyl-2-oxazoline (EtOx) and 2-methoxycarboxyethyl-2-oxazoline

11 (MestOx). Although the direct polymerization seems to be a more straightforward method 12 compared to the here presented two-step hydrolysis-modification method, it should be noted that

13 EtOx is available in bulk quantities while MestOx has to be custom synthesized in a two-step 14 synthetic protocol when starting from methyl succinyl chloride with $57 \%$ overall yield, including 15 double purification by distillation ${ }^{34}$. In this perspective, the demonstrated hydrolysis-modification 16 method may actually be more cost-effective and time-efficient than the direct polymerization 17 method. Moreover, the hydrolysis-modification route is also more versatile as different reactive 18 linkers, such as methyl bromopropionate, methyl acrylate or methyl iso(thio)cyanatopropionate 19 may be used to install similar methyl ester side chains. ${ }^{38}$ 


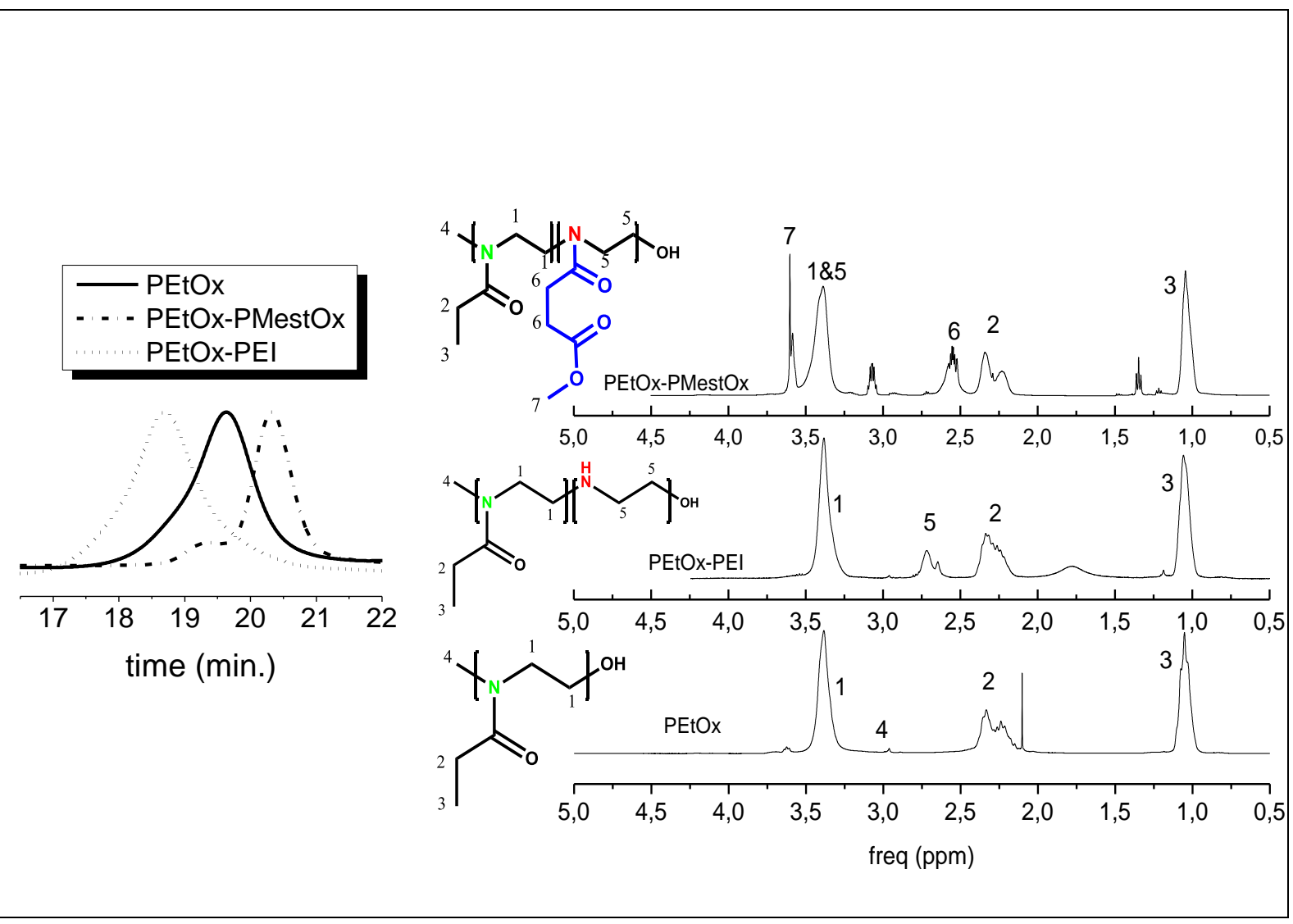

2 Figure 1. Overlay of the SEC traces in HFIP (Left) and ${ }^{1} \mathrm{H}$ NMR spectra of PEtOx, PEtOx-PEI 3 and $\mathrm{PEtOx}-\mathrm{MestOx}$ in $\mathrm{CDCl}_{3}$

6 Post-polymerization modification of PEtOx-MestOx by direct amidation

7 After establishing a robust procedure for the preparation of PEtOx-MestOx, we focused our 8 attention to the amidation of the methyl ester side chains. Even though it is commonly accepted

9 that a direct amidation is not feasible under mild conditions, we did try this out by stirring the

10 PEtOx-MestOx copolymer for two days in neat $n$-propylamine at $70{ }^{\circ} \mathrm{C} .{ }^{1} \mathrm{H}$ NMR spectroscopic

11 analysis of the resulting polymer after precipitation revealed that the methyl ester resonance at 3.7

12 ppm disappeared and a new amide signal appeared at $7.9 \mathrm{ppm}$ indicating successful amidation. 
1 The disappearance of the methylester peaks gives the prove that there is a full conversion from an

2 ester to an amide. In addition, FT-IR also confirmed the successful amidation by disappearance of

3 the methyl ester band at $1730 \mathrm{~cm}^{-1}$ and appearance of a new band at $1531 \mathrm{~cm}^{-1}$ representative for

4 the NH bending in a secondary amide group. Furthermore, SEC analysis in DMA revealed an

5 increase in $\mathrm{M}_{\mathrm{n}}$ from $9.710^{3} \mathrm{Da}$ for PEtOx to $1110^{3}$ for PEtOx-MestOx to $17.810^{3} \mathrm{Da}$ for the $n$ -

6 propyl modified polymer. Importantly, the $Đ$ remained constant at 1.1 indicating that no side

7 reactions take place. Even though it is not fully clear why the direct mild amidation of PEtOx-

8 MestOx is possible, it may be speculated that this is due to the very high concentration and large

9 excess of amine groups, possibly in combination with a neighbouring group effect in which the

10 amide groups attached to the PAOx backbone participate in the reaction mechanism by

11 stabilization of the transition state via the formation of H-bonds.

12 Inspired by the success of this first direct amidation reaction, we continued to explore the scope

13 of this post-polymerization modification reaction for PEtOx-MestOx. Several other readily

14 available and cheap amines, namely aminomethane, aminoethane and hydrazine-hydrate, were

15 utilized using the same Method 1, whereby the reaction is performed in a solution of the amine.

$16{ }^{1} \mathrm{H}$ NMR spectroscopy, FT-IR and SEC again revealed the success of these direct amidation

17 reactions (Table 1). The hydrazine modified polymer is especially interesting as it contains

18 hydrazide side-chains that may be further utilized for conjugation of ketone containing molecules

19 via a pH-degradable hydrazone linker ${ }^{39}$. In a next step, a series of more complex functional amines

20 was explored for direct amidation of PEtOx-MestOx. As these amines are more expensive and

21 often appear as viscous liquids, the reaction conditions were modified resulting in Method 2. In

22 this second method, the polymer is dissolved in acetonitrile and three equivalents of the amine are

23 added. The resulting solution is stirred at $40{ }^{\circ} \mathrm{C}$ overnight. Only if the amine is not soluble in 
1 acetonitrile, the reaction was performed in a similar manner in DMF, which was the case for

2 allylamine and 1-amino-3-butyne. In the case of amino acids such as Leucine ester and Glycine a

3 deprotonation was needed. Therefore basic water was used as a reaction medium. The different

4 tested amines are listed in Table 1 and successful direct amidation was found based on ${ }^{1} \mathrm{H}$ NMR

5 and FT-IR spectroscopy as well as SEC for dimethylamine, Leucine methyl ester, glycine, 1,2-

6 aminoethanol and 1,3-aminopropanol, allylamine, 1-amino-3-butyne, N,N-dimethyl-1,2-

7 ethylenediamine and 1,2-ethylenediamine. As such, we could include a wide variety of functional

8 groups in the side chains of PAOx based on the same PEtOx-MestOx reactive platform. It is

9 important to note, that the reaction with 1,2-ethylenediamin led to a minor increase of $Đ$ and a

10 decrease in $\mathrm{M}_{\mathrm{n}}$. This observation can be ascribed to tailing of the SEC trace due to interaction of

11 the free amine group with the column materials. There is no evidence of double molar mass

12 shoulders indicating that no cross-linking took place. A series of representative ${ }^{1} \mathrm{H}$ NMR spectra,

13 FT-IR spectra and SEC traces of the PEtOx-MestOx and some products after amidation are shown

14 in Figure 2, 3 and 4, respectively. The ${ }^{1} \mathrm{H}-\mathrm{NMR}$ spectra clearly show that the peak at $3.7 \mathrm{ppm}$ of

15 the methyl ester group in PEtOx-PMestOx disappears upon amidation. There is also a notable

16 change of signal 6 , which after reaction with the amine moves closer to, and partially overlap with

17 the peak around $2.2 \mathrm{ppm}$. These peaks merge together because of the similarity between the side

18 chain of the PEtOX and the side chain of the succinyl. FT-IR clearly shows the disappearance of

19 the band at $1730 \mathrm{~cm}^{-1}$ of the methyl ester carbonyl and reveals the appearance of a new band in

20 the region between $1530-1560 \mathrm{~cm}^{-1}$ a finger print of the NH bending.. Finally, SEC analysis clearly

21 shows that the polymer molar mass distributions have very similar shape before and after

22 amidation demonstrating that no polymer coupling occurred. 

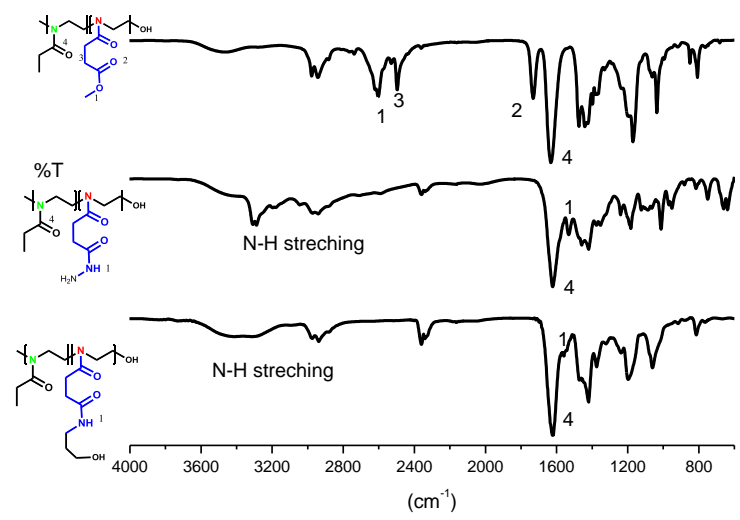

2 Figure 2: FT-IR data starting from the top with the PEtOx-MestOx and below two

3 examples of polymers after the direct amidation

5 Table 1: Overview of the direct amidation of PEtOx-MestOx with various amines.

\begin{tabular}{|c|c|c|c|c|c|}
\hline & $R_{1}$-group & \begin{tabular}{|c|} 
Chemical shift \\
amide proton(ppm) ${ }^{1}$
\end{tabular} & $\mathrm{Mn}(\mathrm{kDa})^{2}$ & $\mathbf{D}^{2}$ & Method $^{3}$ \\
\hline aminomethane & $\mathrm{H}_{2} \mathrm{~N}-\mathrm{CH}_{3}$ & 6.4 & 17.7 & 1.1 & 1 \\
\hline aminoethane & & 6.5 & 17.7 & 1.1 & 1 \\
\hline $\begin{array}{l}\text { 1-Amino } \\
\text { propane }\end{array}$ & & 7.9 & 17.8 & 1.1 & 1 \\
\hline dimethylamine & & 6.3 & 17.8 & 1.1 & 2(water) \\
\hline hydrazine monohydrate & $\mathrm{NH}_{2}$ & 9 & 17.7 & 1.1 & 1 \\
\hline leucine methylester & & $\mathrm{nd}^{4}$ & 18 & 1.1 & 2(basic water) \\
\hline glycine & & $\mathrm{nd}^{4}$ & 18 & 1.1 & 2(basic water) \\
\hline 2-amino-1-ethanol & & nd 4 & 22 & 1.1 & 2(acetonitrile) \\
\hline 3-amino-1-propene & & $\mathrm{nd}^{4}$ & 16.5 & 1.1 & 2(DMF) \\
\hline 1-amino-3-butyne & & 7.8 & 16.7 & 1.1 & $2(\mathrm{DMF})$ \\
\hline
\end{tabular}




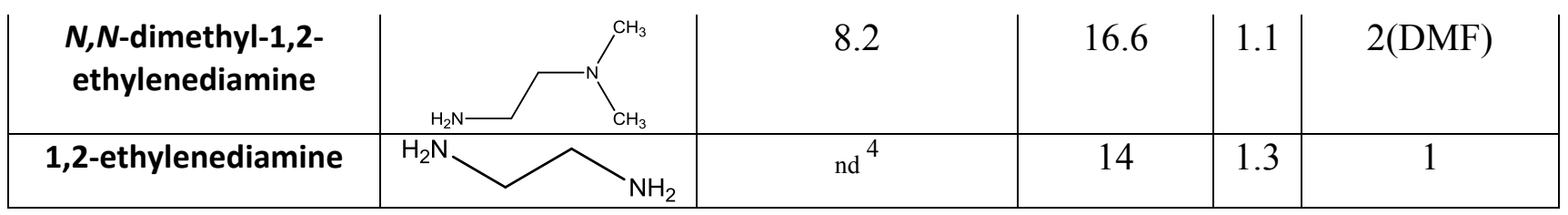

1

$2 \quad{ }^{1}$ Chemical shift in ${ }^{1} \mathrm{H}-\mathrm{NMR}$ spectra recorded in $\mathrm{CDCl}_{3}$.

$3 \quad{ }^{2}$ Calculated from SEC analysis with DMA as eluent and PMMA as calibration.

$4 \quad{ }^{3}$ Method 1: amine is used as reaction solution; method 2: The amidation reaction is done in another

5 solvent specified between brackets.

$6 \quad{ }^{4}$ Not distinguishable as the ${ }^{1} \mathrm{H}-\mathrm{NMR}$-spectra were recorded in DMSO and the NH peak could not

7 be seen.

8
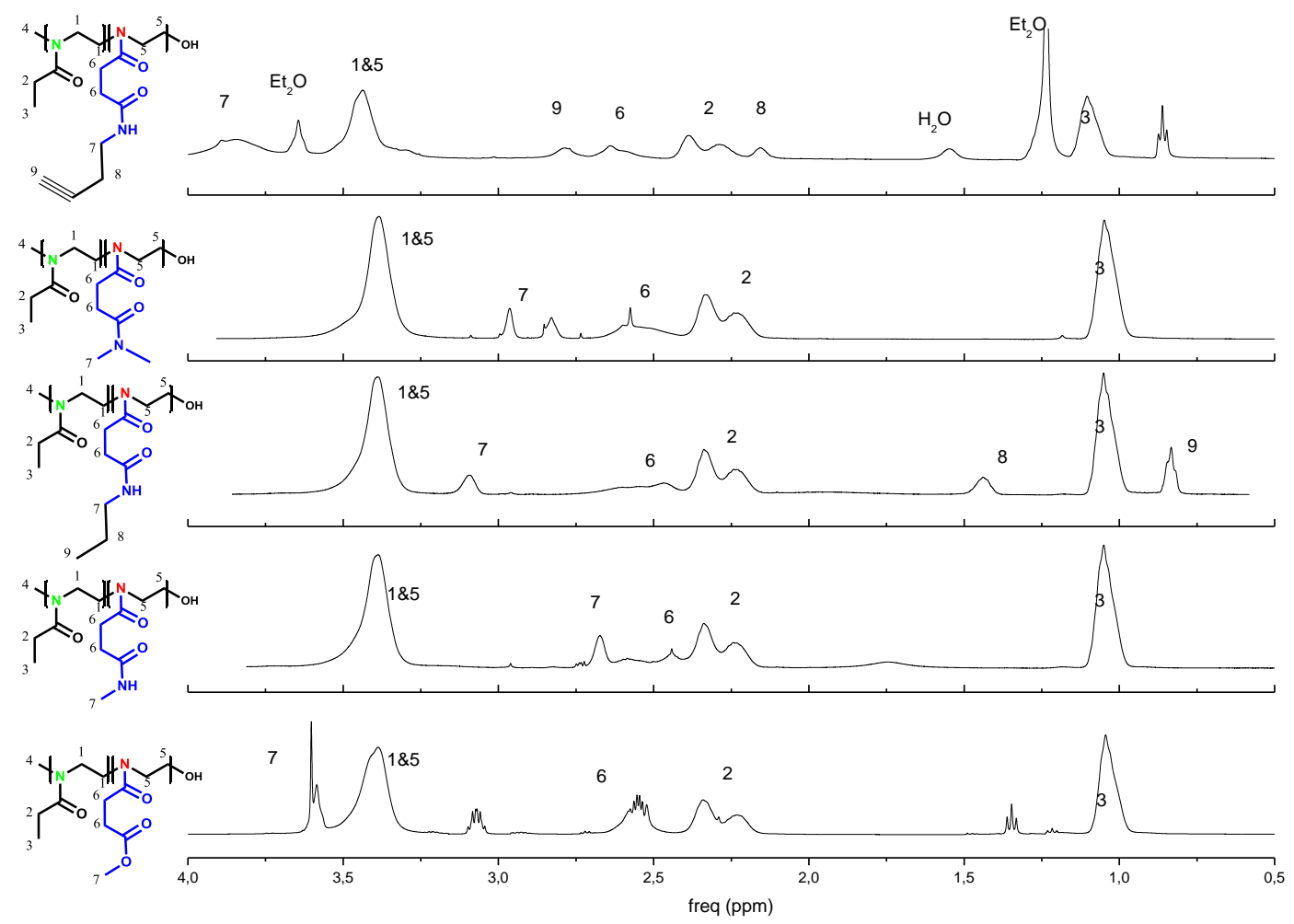
1 Figure 3: Overlay of the ${ }^{1} \mathrm{H}-\mathrm{NMR}$ spectra of PEtOx-MestOx and four products after direct 2 amidation with different amines.

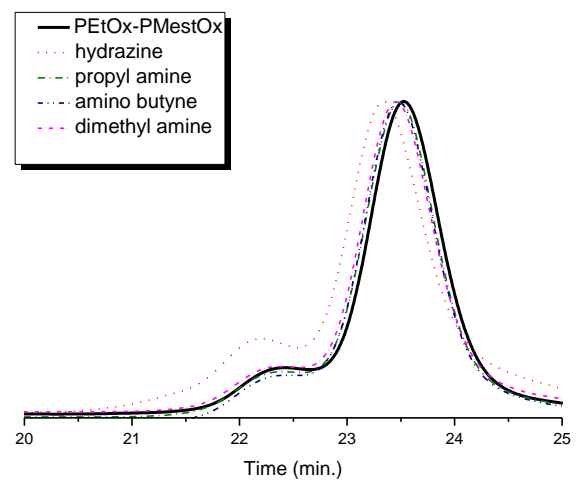

4 Figure 4: Overlay of normalized SEC (HFIP)traces of PEtOx-PMestOx and four examples of 5 amidated polymers functionalized with hydrazine, allyl amine, 1-amino-3-butyne, cystamine

6 To show that the direct amidation is also possible on homopolymers, PMestOx with DP 20 was 7 synthesized by CROP of MestOx using methyl tosylate as initiator. This resulting polymer was 8 analyzed by ${ }^{1} \mathrm{H}-\mathrm{NMR}$ spectroscopy, MALDI-TOF MS and SEC revealing the formation of a

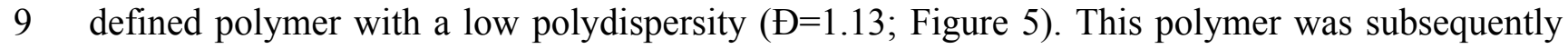
10 modified with 1-aminopropane via method 1 (bulk method) by dissolving the polymer in 111 aminopropane and stirring overnight at $37^{\circ} \mathrm{C}$. The MALDI-TOF mass spectrum of the resulting 12 polymer clearly shows that there is a shift in mass in comparison to the begin product (Figure 5).

13 MALDI-TOF MS of the PMestOx revealed the expected spacing of $157 \mathrm{~m} / \mathrm{z}$ corresponding to the 14 MestOx monomer while after reaction the spacing increased to $184.24 \mathrm{~m} / \mathrm{z}$ corresponding to the 15 amidopropane side chain. Furthermore, the exact mass of $3197 \mathrm{~m} / \mathrm{z}$ for PMestOx corresponds to a 16 polymer with 20 repeat units and methyl and $\mathrm{OH}$ end-groups charged with a sodium cation. After 17 the amidation reaction, the exact mass of 3738 is found corresponding to the amido-propane 
1 functionalized polymer with 20 repeat units and methyl and $\mathrm{OH}$ end-groups and charged with a

2 sodium cation. These results confirm a clean amidation of all methyl ester side chains into. The

$3 \quad{ }^{1} \mathrm{H}-\mathrm{NMR}$ spectra also demonstrate that the methyl ester groups disappeared after amidation while

43 new peaks appeared corresponding to the propyl side chains (signals 4-6 in Figure 5). Finally,

5 SEC confirmed an increase in hydrodynamic volume after the amidation reaction while the $Ð$

6 mildly increased from 1.13 to 1.17 , albeit no clear shoulders are observable (Figure 5).
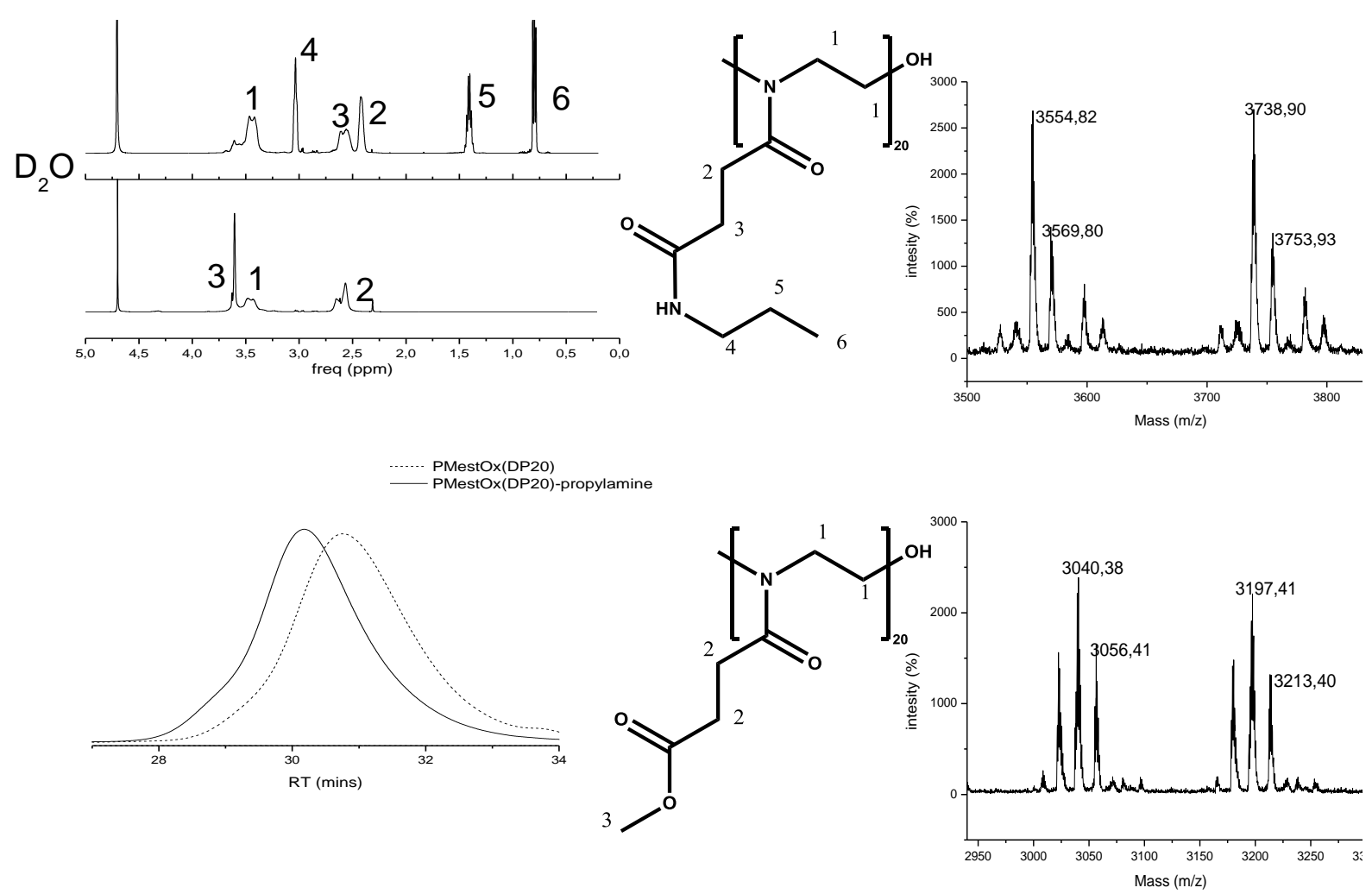

8 Figure 5: Overview of the ${ }^{1} \mathrm{H}-\mathrm{NMR}$ data in $\mathrm{D}_{2} \mathrm{O}$ (top left), MALDI-TOF MS (right) and SEC

9 (DMA as eluent; bottom left) analysis of the pure PMestOx with 20 repeating units before and

10 after amidation with 1-aminopropane. The bottom ${ }^{1} \mathrm{H}$ NMR and MALDI TOF mass spectra show

11 PMestOx and the top spectra show the polymer after amidation with propylamine. 


\section{Conclusion and outlook}

4 We successfully showed a novel modification procedure to expand the chemical toolbox for

5 poly(2-oxazoline)s PEtOx. Starting from the controlled hydrolysis of well-defined PEtOx a

6 copolymer is obtained with a specific amount of amine units to control the number of functional

7 groups that can be incorporated. These amine units are subsequently reacted with methyl succinyl

8 chloride restoring the poly(2-oxazoline) side chain structure while installing a methyl ester

9 functionality. Even though such methyl ester functionalized copolymers can also be obtained via

10 a direct polymerization method, the post polymerization modification route is, arguably, more cost

11 effective and less time consuming.

12 The copoly(2-oxazoline)s containing methyl ester side chains methyl ester group is

13 demonstrated to undergo a direct amidation reaction, enabling the installation of a wide-range of

14 side chain amide groups and functionalities. An important feature is that these reactions efficiently

15 proceeds without a catalyst, proposedly due to activation by neighbouring amide groups.

16 Furthermore, we also demonstrated the synthesis of a PMestOx homopolymer with 20 repeat units

17 by direct CROP of MestOx. The direct amidation of this model polymer with 1-aminopropane

18 clearly revealed that all methyl ester side-chains can be modified.

19 We are confident that this direct amidation side-chain modification approach will be an

20 important extension of the PAOx toolbox that will lead to the development of novel applications

21 of these polymers.

23 Acknowledgements. RH thanks IWT for support via the Strategic Basic Research program (SBO-

24 120049). MM and RH acknowledge the Special Research Fund of Ghent University. 


\section{References}

1. Tomalia, D. a. \& Sheetz, D. P. Homopolymerization of 2-alkyl- and 2-aryl-2-oxazolines. J. Polym. Sci. Part A-1 Polym. Chem. 4, 2253-2265 (1966).

2. Seeliger, W. et al. Recent syntheses and reactions of cyclic imidic esters. Angew. Chem. Int. Ed. Engl. 5, 875-88 (1966).

3. Kagiya T, F. K. Ring-opening polymerization of 2-substituted 2-oxazolines. Polym. Lett. 4, 441-445 (1966).

4. Levy A,Bassiri, L. Polymerization of cyclic iminoethers. J.Polym.Sci.PartB:Polym.Lett 5, 2253-2265 (1967).

5. Aoi, K. \& Okada, M. Polymerization of oxazolines. Prog. Polym. Sci. 21, 151-208 (1996).

6. Kobayashi, S. Ethylenimine polymers. Prog. Polym. Sci. 15, 751-823 (1990).

7. Hoogenboom, R. Poly(2-oxazoline)s: a polymer class with numerous potential applications. Angew. Chem. Int. Ed. Engl. 48, 7978-94 (2009).

8. Adams, N. \& Schubert, U. S. Poly(2-oxazolines) in biological and biomedical application contexts. Adv. Drug Deliv. Rev. 59, 1504-20 (2007).

9. Schlaad, H. \& Hoogenboom, R. Poly(2-oxazoline)s and related pseudo-polypeptides. Macromol. Rapid Commun. 33, 1599 (2012).

10. Luxenhofer, R. et al. Poly(2-oxazoline)s as polymer therapeutics. Macromol. Rapid Commun. 33, 1613-31 (2012).

11. Hoogenboom, R., Paulus, R. M., Pilotti, A. \& Schubert, U. S. Scale-up of MicrowaveAssisted Polymerizations in Batch Mode: The Cationic Ring-Opening Polymerization of 2-Ethyl-2-oxazoline. Macromol. Rapid Commun. 27, 1556-1560 (2006).

12. Kempe, K., Lobert, M., Hoogenboom, R. \& Schubert, U. S. Screening the synthesis of 2substituted-2-oxazolines. J. Comb. Chem. 11, 274-80 (2009).

13. Kjøniksen, Anna-lena, H. R. Thermoresponsive Poly(2-oxazoline) Block Copolymers Exhibiting Two Cloud Points: Complex Multistep Assembly Behavior. Macromolecules 45, 4337-4345 (2012). 
14. Persigehl, P., Jordan, R. \& Nuyken, O. Functionalization of amphiphilic poly(2-oxazoline) block copolymers: A novel class of macroligands for micellar catalysis. Macromolecules 33, 6977-6981 (2000).

15. Milonaki, Y., Kaditi, E., Pispas, S. \& Demetzos, C. Amphiphilic gradient copolymers of 2-methyl- and 2-phenyl-2-oxazoline: self-organization in aqueous media and drug encapsulation. J. Polym. Sci. Part A Polym. Chem. 50, 1226-1237 (2012).

16. Fetsch, C. \& Luxenhofer, R. Highly Defi ned Multiblock Copolypeptoids : Pushing the Limits of Living Nucleophilic. Macromol. Rapid Commun. 33, 1708-1713 (2012).

17. Guillerm, B., Monge, S., Lapinte, V. \& Robin, J.-J. How to modulate the chemical structure of polyoxazolines by appropriate functionalization. Macromol. Rapid Commun. 33, 1600-12 (2012).

18. Verbraeken, B. K. H. R. in Encycl. Polym. Sci. Technol. 1-39 (2014).

19. Rossegger, E., Schenk, V. \& Wiesbrock, F. Design Strategies for Functionalized Poly(2oxazoline)s and Derived Materials. Polymers (Basel). 5, 956-1011 (2013).

20. Huntsville, J. M., Huntsville, M. D., Madison, K., Madison, Z. \& Maria, F. Activated polyoxazolines and compositions comprising the same. 7943141, 1-44 (2011).

21. Knop, K., Hoogenboom, R., Fischer, D. \& Schubert, U. S. Poly(ethylene glycol) in drug delivery: pros and cons as well as potential alternatives. Angew. Chem. Int. Ed. Engl. 49, 6288-308 (2010).

22. Barz, M., Luxenhofer, R., Zentel, R. \& Vicent, M. J. Overcoming the PEG-addiction: well-defined alternatives to PEG, from structure-property relationships to better defined therapeutics. Polym. Chem. 2, 1900 (2011).

23. De la Rosa, V. R., Bauwens, E., Monnery, B. D., De Geest, B. G. \& Hoogenboom, R. Fast and accurate partial hydrolysis of poly(2-ethyl-2-oxazoline) into tailored linear polyethylenimine copolymers. Polym. Chem. (2014). doi:10.1039/c4py00355a

24. Sedlacek, O., Monnery, B. D., Filippov, S. K., Hoogenboom, R. \& Hruby, M. Poly(2oxazoline)s--are they more advantageous for biomedical applications than other polymers? Macromol. Rapid Commun. 33, 1648-62 (2012).

25. Kempe, K., Hoogenboom, R., Jaeger, M. \& Schubert, U. S. Three-Fold Metal-Free Efficient ('Click') Reactions onto a Multifunctional Poly(2-oxazoline) Designer Scaffold. Macromolecules 44, 6424-6432 (2011).

26. Mais, U., Binder, W. H., Knaus, S. \& Gruber, H. Synthesis and 13 C CP MAS NMR spectroscopy of cellulose- graft -poly ( N -acetylethylenimine ). 2115-2122 (2000). 
1 27. Taubmann, C., Luxenhofer, R., Cesana, S. \& Jordan, R. First aldehyde-functionalized

2 poly(2-oxazoline)s for chemoselective ligation. Macromol. Biosci. 5, 603-12 (2005).

3 28. Legros, C., De Pauw-Gillet, M.-C., Tam, K. C., Lecommandoux, S. \& Taton, D.

Aldehyde-functional copolymers based on poly(2-oxazoline) for post-polymerization modification. Eur. Polym. J. 62, 322-330 (2015).

29. Diehl, C. \& Schlaad, H. Thermo-responsive polyoxazolines with widely tuneable LCST. Macromol. Biosci. 9, 157-61 (2009).

30. Englert, C. et al. Linear Poly(ethylene imine)-Based Hydrogels for E ff ective Binding and Release of DNA. 2-9 (2014).

10 31. Kempe, K. et al. Responsive glyco-poly(2-oxazoline)s: synthesis, cloud point tuning, and lectin binding. Biomacromolecules 12, 2591-600 (2011).

32. Luxenhofer, R. \& Jordan, R. Click Chemistry with Poly(2-oxazoline)s. Macromolecules 39, 3509-3516 (2006).

33. Lava, K., Verbraeken, B. \& Hoogenboom, R. Poly (2-oxazoline ) s and click chemistry : a versatile toolbox towards multi-functional polymers. Eur. Polym. J. In press, 1-29 (2015).

34. Bouten, P. J. M. et al. Accelerated living cationic ring-opening polymerization of a methyl ester functionalized 2-oxazoline monomer. Polym. Chem. 6, 514-518 (2014).

35. Van Kuringen, H. P. C. et al. Partial hydrolysis of poly(2-ethyl-2-oxazoline) and potential implications for biomedical applications? Macromol. Biosci. 12, 1114-23 (2012).

38. Hoogenboom, R. Polyoxazoline polymers and methods for their preparation, conjugates of

36. Lambermont-Thijs, H. M. L. et al. Linear Poly(ethylene imine)s by Acidic Hydrolysis of Poly(2-oxazoline)s: Kinetic Screening, Thermal Properties, and Temperature-Induced Solubility Transitions. Macromolecules 43, 927-933 (2010).

37. Jäger, M., Schubert, S., Ochrimenko, S., Fischer, D. \& Schubert, U. S. Branched and linear poly(ethylene imine)-based conjugates: synthetic modification, characterization, and application. Chem. Soc. Rev. 41, 4755-67 (2012). 
1 Include Graphical abstract here, see guidelines macromolecules

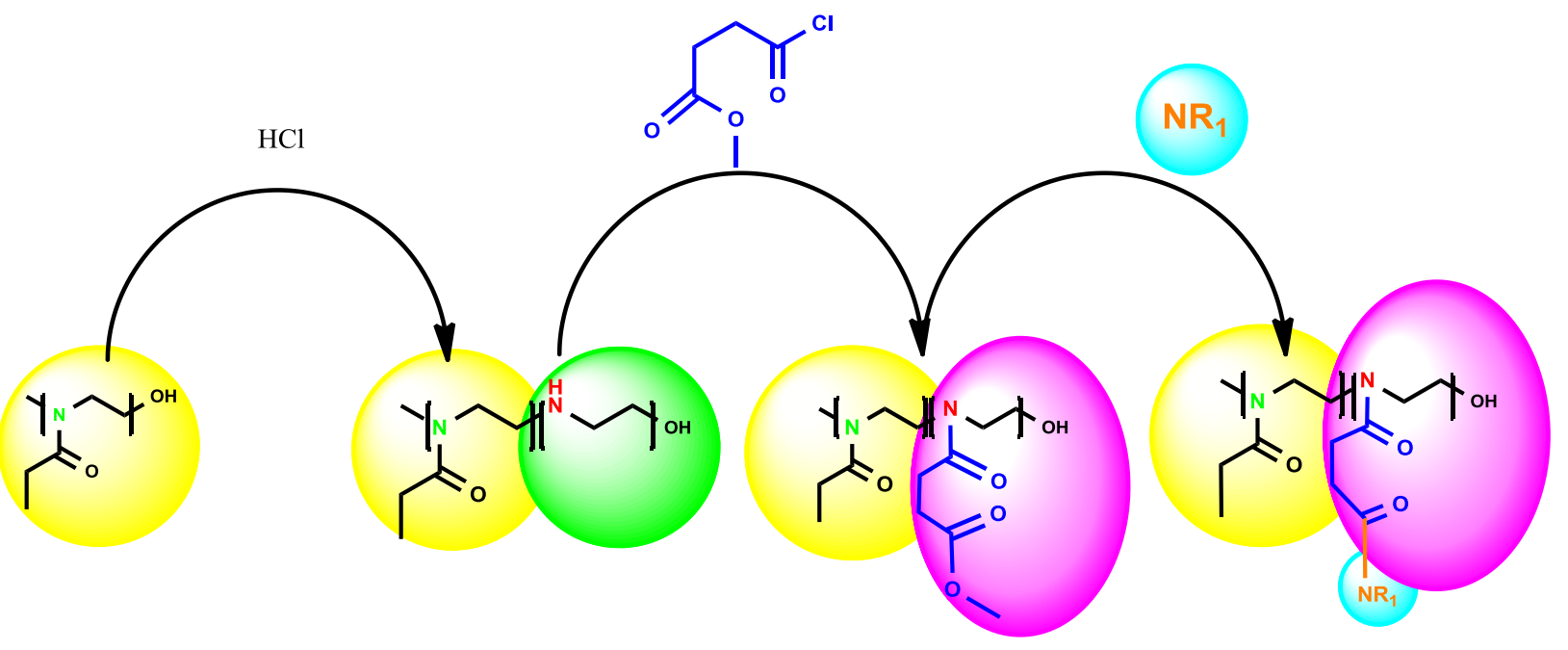

